\title{
Time Equals Brain - Retrospective Analysis of Thrombolysis in Regional Australia to Determine Factors which Influence Door to Needle Time
}

\author{
Udit Nindra*1, Toni Marie Wonson ${ }^{2}$ and Karen Fuller ${ }^{3}$ \\ ${ }^{1}$ Resident Medical Officer, Wollongong Hospital, Australia \\ ${ }^{2}$ Stroke Clinical Nurse Consultant, Wollongong Hospital, Australia \\ ${ }^{3}$ Staff Specialist, Neurology, Wollongong Hospital, Australia
}

*Corresponding author: Udit Nindra, 1Resident Medical Officer, Wollongong Hospital, Australia.

\begin{abstract}
A 3-year retrospective cohort analysis of all acute stroke admissions in Wollongong Hospital, a major regional referral centre in New South Wales, was completed to determine the causes of in-hospital delays for thrombolysis. Data collected included age, baseline National Institute of Health Stroke Scale (NIHSS) score, onset time, arrival time, CT imaging \& reporting time and outcomes of the event. From 656 admissions, 70 cases of thrombolysis and 56 cases of endovascular thrombectomy (ECR) were recorded. The mean time from onset to arrival was 85 minutes, from arrival to CT was 31 minutes and from door to needle time (DTN) was 108 minutes. Multiple regression analysis revealed an inverse linear association between onset to arrival time and DTN. For every 30-minute delay in hospital arrival, there was a 13-minute reduction in DTN. Age, stroke severity and gender were not shown to impact treatment times. The results showed that there was a paradoxical association between arrival time and DTN. The cause for this was not clearly appreciable but, similar to previous studies, was likely due to a lack of urgency when initiating management when patients arrived sooner after symptom onset. In light of this, a pilot education protocol to promote the motto 'time equals brain' amongst stroke first responders is being implemented to aim to reduce DTN to less than 80 minutes in the Illawarra region.
\end{abstract}

\section{Introduction}

In 2012, an estimated 377,000 people had a stroke at some time in their lives. $71 \%$ of these patients were aged 65 and over $[1,2]$. Treatments for stroke have rapidly progressed with intravenous thrombolysis and endovascular thrombectomy (ECR) being mainstays of current acute therapy. Acute treatment of stroke is time dependent. Current Australian guidelines for IT \& ECR are varied depending on region and practitioner. However the consensus is that for large vessel strokes, IT should be considered if patients present within 4.5 hours of symptom onset and ECR should be completed within 24 hours of symptom onset [3]. In the Illawarra region, patients eligible for ECR must be transferred to quaternary centers for intervention. The purpose of this study is to outline barriers to timely thrombolysis and early referral to ECR in regional Australia and determine factors that influence door to needle time (DTN) and arrival to referral time (ATR).

\section{Methods}

Wollongong Hospital is a 500-bed hospital with a catchment area that extends for over 250 kilometres along the southern region of New South Wales. The hospital receives the vast majority of patients formally diagnosed with strokes in the area with transfers from seven peripheral hospitals in the district. According to the 2015 Australian Bureau of Statistics database there are approximately 300,067 people living in the Illawarra region with an additional 147,960 patients in the Shoalhaven region. This accumulates to a total of 448,027 people in Wollongong Hospital's catchment area [4]. Based on local protocol, all patients admitted to the stroke unit at Wollongong Hospital have de-identified data collected and stored on centralised databases that include diagnosis, treatment and outcomes. A manual search through this database for all patients with ischemic strokes was performed for 
cases admitted between March 2016 and October 2018. This time period was chosen as the local data collector had changed prior to March 2016 and there was significant inconsistency prior data. As the data was also de-identified, backtracking was not possible.

A total of 656 patients with a diagnosis of non-haemorrhagic / possible ischemic stroke were admitted to Wollongong Hospital over this period based on the inclusion/exclusion criteria outlined in Table 1. Baseline CT Brain imaging was conducted in

Table 1: Inclusion \& Exclusion Criteria for Participants.

\begin{tabular}{|c|c|}
\hline Inclusion Criteria \\
\hline$\bullet$ & $\begin{array}{l}\text { Admission to Wollongong hospital as a probable ischemic } \\
\text { stroke }\end{array}$ \\
\hline$\bullet$ & Formal diagnosis of ischemic stroke post imaging \\
\hline$\bullet$ & $\begin{array}{l}\text { Intravenous thrombolysis and/or endovascular thrombecto- } \\
\text { my completed }\end{array}$ \\
\hline Exclusion Criteria \\
\hline$>$ & $\begin{array}{l}\text { Stroke-mimics that are diagnosed in clinically or subsequent- } \\
\text { ly post imaging }\end{array}$ \\
\hline$>$ & $\begin{array}{l}\text { Haemorrhagic stroke on initial CT or subsequent MRI imaging } \\
\text { diagnosed }\end{array}$ \\
\hline$>\quad \begin{array}{l}\text { No formal diagnosis of ischemic stroke including those diag- } \\
\text { nosed with Transient Ischemic Attacks }\end{array}$ \\
\hline
\end{tabular}

\section{Results}

Of the 656 patients diagnosed with ischemic strokes over the 2-year period, 70 cases underwent IT. The demographics of the patients are shown in Table 2. The mean age of these patients was 62.9 years with majority male. IT is commenced onsite, typically post transfer of the patient into the stroke unit. ECR however, requires transfer to a referral centre with neurointerventionalist support, which occurs via road-ambulance. ECR was completed in 56 patients over the time period. From this small cohort, 6 patients underwent IT followed by ECR which are summarized in Table 3 (Table 2 \& 3).

Table 2: Patient Demographics for those undergoing thrombectomy or thrombolysis.

\begin{tabular}{|c|c|}
\hline Thrombolysed Patients & \\
\hline Mean Age: & 62.9 years \\
\hline Median Age: & 65.5 years \\
\hline Male: & $27(54 \%)$ \\
\hline Female: & $23(46 \%)$ \\
\hline Mean Initial NIHSS1 Score & $8.9(1-21)$ \\
\hline Mean Post-procedure NIHSS1 Score & $4.8(0-31)$ \\
\hline Endovascular Thombectomy Patients & 68.7 years \\
\hline Mean Age: & 69.8 years \\
\hline Median Age: & $26(55 \%)$ \\
\hline Male: & $21(45 \%)$ \\
\hline Female: & $12.6(1-29)$ \\
\hline Mean Initial NIHSS1 Score & $3.6(0-16)$ \\
\hline Mean Post-procedure NIHSS1 Score & \\
\hline
\end{tabular}

1: NIHSS (National Institute of Health and Stroke Safety
$98 \%$ of patients with exclusions including palliation or complete symptom resolution. CT angiogram imaging however was done based on the clinician's judgement regarding possible suitability for endovascular thrombectomy or concern regarding secondary vascular pathology such as dissection or aneurysm. A manual check of all patients was then done to determine those who underwent intravenous thrombolysis and/or endovascular thrombectomy (Table 1).

Table 3: Summary of six cases that underwent thrombolysis and thrombectomy.

\begin{tabular}{|c|c|c|c|c|c|}
\hline Patient & Age & Gender & $\begin{array}{c}\text { Initial } \\
\text { NIHSS }\end{array}$ & $\begin{array}{c}\text { Onset } \\
\text { time* to } \\
\text { Thrombolysis }\end{array}$ & $\begin{array}{c}\text { Onset } \\
\text { Time* to } \\
\text { Thombectomy }\end{array}$ \\
\hline 1 & 59 & F & 12 & $2 \mathrm{~h} 25 \mathrm{~m}$ & $4 \mathrm{~h} 31 \mathrm{~m}$ \\
\hline 2 & 72 & $\mathrm{~F}$ & 10 & $4 \mathrm{~h} 04 \mathrm{~m}$ & $6 \mathrm{~h} 27 \mathrm{~m}$ \\
\hline 3 & 86 & $\mathrm{~F}$ & 7 & $4 \mathrm{~h} 20 \mathrm{~m}$ & $7 \mathrm{~h} 40 \mathrm{~m}$ \\
\hline 4 & 23 & M & 3 & $3 \mathrm{~h} 00 \mathrm{~m}$ & $5 \mathrm{~h} 20 \mathrm{~m}$ \\
\hline 5 & 45 & M & 9 & $2 \mathrm{~h} 45 \mathrm{~m}$ & $4 \mathrm{~h} 29 \mathrm{~m}$ \\
\hline 6 & 66 & M & 19 & $3 \mathrm{~h} 15 \mathrm{~m}$ & $5 \mathrm{~h} 01 \mathrm{~m}$ \\
\hline
\end{tabular}

*Onset time is defined as the time the patient was last seen well or the known time of onset as per bystanders who witnessed the event

The mean onset to needle time for IT was 3 hours 13 minutes (range between 2 to 4.5 hours). No thrombolysis attempts were made outside the 4.5-hour time window. The mean initial National Institute of Health and Stroke Safety (NIHSS) score was 8.9 compared with a mean post thrombolysis score of 4.8. There were two cases of an increase in the stroke severity score, both of which were the result of intracranial haemorrhage. The mean DTN was 108 minutes.

The mean age for those undergoing ECR was 68.7 years. The mean initial NIHSS score was 12.6 compared with a median postthrombectomy NIHSS score of 3.6. There were no cases of measured increase in the stroke severity score. However, the recording of the post-operative NIHSS score was only completed in 19\% of patients. Furthermore, there were three instances of intra-operative complications including unsuccessful stenting and intra-cerebral haemorrhage. The mean ART was 150 minutes.

Univariate analysis was completed between DTN \& ATR and other potential variables including baselines NIHSS score, age, gender, arrival-to-CT time and onset to arrival time. From this, there was no correlation between DTN or ATR with age $(p=0.70, p$ $=0.67)$, baseline NIHSS ( $p=0.90, p=0.71)$ and gender $(p=0.46, p$ $=0.44$ ). However, a number of clinically important associations are shown in figures 1 to 4 below.

The mean time from onset to arrival was 85 minutes and from arrival to CT was 31 minutes. Figure 1 demonstrates that using univariate analyses, a negative statistically significant correlation is seen between DTN and onset to arrival time as well as arrival to CT Time. Multiple regression analysis maintained these inverse linear associations. For every 30-minute delay in hospital arrival, there was a 13-minute reduction in DTN $(\mathrm{F}=7.68, \mathrm{p}<0.01)$. Conversely, 
in Figure 2, a positive correlation was seen between DTN and arrival to CT Time on univariate $(\mathrm{p}<0.01)$ and multivariate $(\mathrm{F}=5.08$, $\mathrm{p}=0.03$ ) analysis. For every 10 minute delay in CT imaging, there was a 6-minute delay in DTN (95\% CI 2-11 minutes).
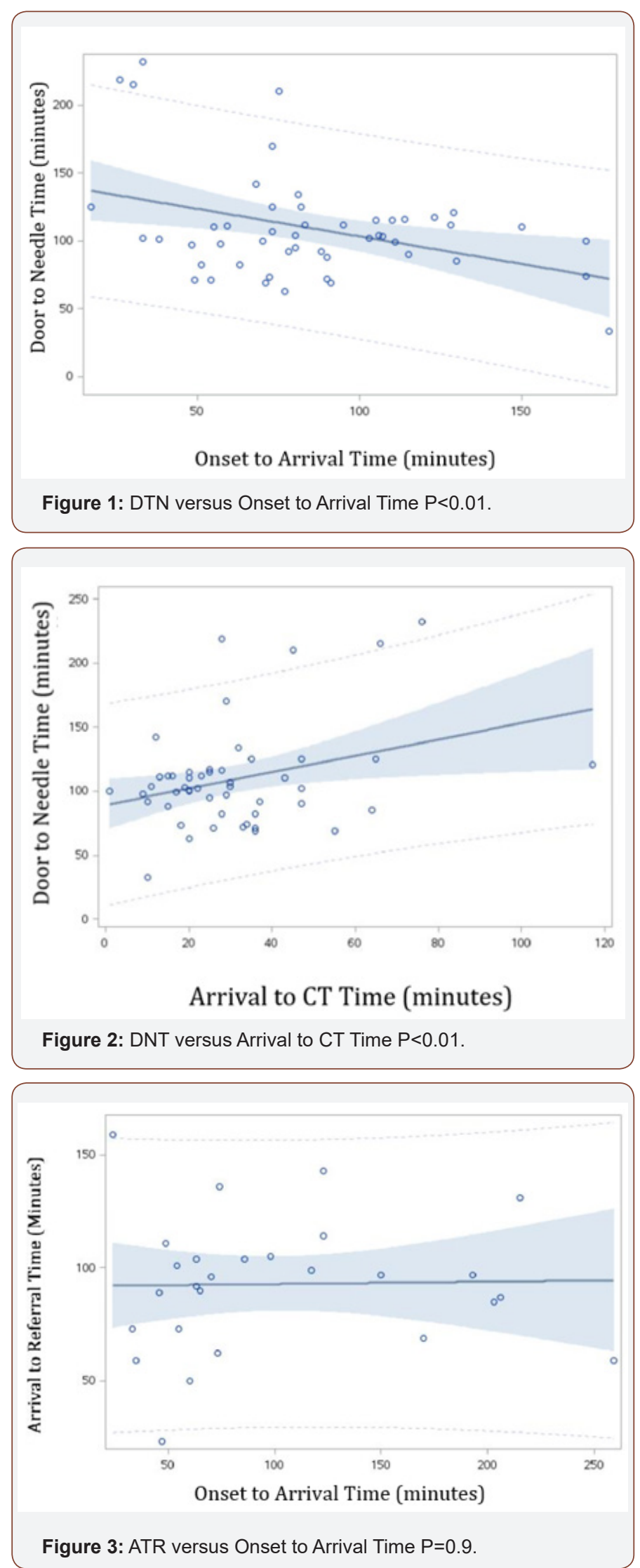

When analysing arrival to referral time, there was no clear association between ART and onset to arrival time, as shown in
Figure $3(\mathrm{p}=0.9)$. However, in Figure 4, ART was shown to correlate positively with arrival to CT Time $(\mathrm{p}=0.02)$. For every 10 -minute delay in CT imaging, there was a 9-minute delay in ART (95\% CI 1-16 minutes).

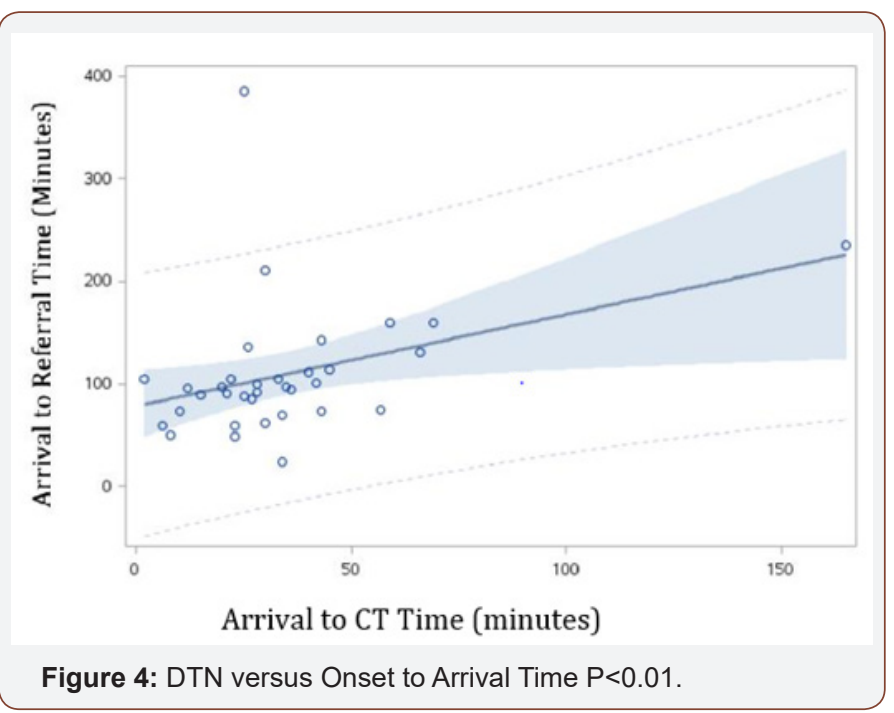

\section{Discussion}

\section{Intravenous thrombolysis}

Our results show that the only two variables with statistically significant associations with DTN were onset to arrival time and arrival to CT time. The latter is an expected finding as significant attempts have been made within primary stroke centres to expedite imaging including direct ambulance drop off to CT scanners, mobilisation of stroke teams and even mobile ambulatory CT devices in Victoria. The paradoxical nature of early onset to arrival time leading to delayed DTN has also been previously documented [5-7]. For example, the STARS study [6], which was a prospective, multicentre trial involving nearly 400 patients showed that for every 30 -minute delay between the onset of stroke and arrival to hospital, there was an associated 15-minute decrease in the time taken to initiation intravenous thrombolysis. These results are similar to those found by this retrospective cohort analysis. A further study completed in Miami [7] also commented on this paradoxical nature of delay in thrombolysis but was not able to accurately delineate the cause for such. One theory was that there was a subjective lack of urgency created by the NIHSS guidelines for a 4.5 hour thrombolysis window. This meant that that those patients who present earlier, provided a less urgent situation for doctors, hence allowing 'more time' to decide for thrombolysis as the initiation would remain within such window.

It is known, however, that acute stroke management is timesensitive. Intravenous thrombolysis is more beneficial the earlier it is initiated with studies showing that thrombolysis initiated within 90 minutes of symptom onset has twice the benefit as between 90 minutes and three hours of onset [8]. Hence there is a possibly that this paradoxical delay in treatment is causing worsening patient outcomes but the extent to which this is, is not possible to determine. 
In 2017, the Stroke Foundation released its annual report on the state of stroke services throughout Australia comparing a number of parameters including stroke treatment, response times, use of IT, ECR and imaging [5]. Annually, the Illawarra region receives approximately 223 stroke admissions, marginally higher than the national \& New South Wales medians of 190 and 220 respectively. Nationally, amongst 127 hospitals and over 31,000 acute stroke admissions, $36 \%$ of patients arrived at hospital within the 4.5 -hour time window for thrombolysis. $13 \%$ of these patients would then undergo IT with only $30 \%$ of cases occurring within 60 minutes of arriving at hospital. Nationally, the mean time between onset and IT was 2 hours 36 minutes [5]. In comparison, for patients in the Illawarra Shoalhaven Local Health District (ISLHD), 59\% arrived within the 4.5-hour thrombolysis window. Of these patients, $19 \%$ underwent IT but only one case occurred within 60 minutes of arrival to hospital.

\section{Endovascular thrombectomy}

As neurointerventional treatment is not currently available in the Illawarra region, transfer to quaternity centres are required. Our study shows that delays in CT imaging leads to delays in ART but onset to arrival time does not. For the majority of patients in the Illawarra region a completed CT Brain including angiography report would be available within 1 hour of the patient's arrival. Although CT reporting is not mandatory for experienced clinicians, in such a regional setting, the responding doctor to the stroke call may not always have this expertise and the Neurologist on-call may not be able to directly access the images. Despite this, our study results showed that there was an additional 48-minute delay after CT reporting for referral to be made and the reasons for such are unclear.

Delays in ECR lead to worsening outcomes for patients. One study has shown that symptom onset to reperfusion time of 150 minutes led to $91 \%$ estimated probability of functional independence, which decreased by $10 \%$ over the next hour and by $20 \%$ with every subsequent hour of delay $[9,10]$. Thus, by improving more rapid access to imaging will undoubtedly improve patient outcomes. From a regional perspective, previous studies have shown that inter-hospital delays for ECR are associated with significant treatment delays and lower chance of a good outcome [11]. Hence, there may be an increased need for more neurointerventionalists at regional centres such as in the Illawarra as well.

\section{Future direction}

Currently, in order to counteract this paradoxical result, a clinical education trial is being implemented within the Wollongong district that involves registrars recognising the data that highlights that clinicians often show a lack of urgency when it comes to acute stroke management. The education sessions are run by Neurologists who stress the importance of the motto 'Time equals brain.' This trial will be completed in mid-2019 to assess if education and selfrecognition of behaviour can result an improvement in DTN in acute stroke. The aim of the trial is to reduce the mean DNT to less than 80 minutes.

\section{Conclusion}

Currently, compared nationally, there are is a greater proportion of patients who are presenting with stroke like symptoms amenable to acute stroke management with intravenous thrombolysis and/or endovascular thrombectomy in the Illawarra region. Although these patients are receiving appropriate therapy, there are significant delays to treatment leading to treatment times that fall below the Australian average. Further policies and improved cohesion between hospital teams is required to improve these results. Acute stroke management is time dependent, and with these improvements, clinical outcomes for patients are likely to improve.

\section{Data Availability}

For data availability, please contact author at udit.nindra@ health.nsw.gov.au

\section{Conflicts of Interest}

Nil.

\section{Funding}

Nil.

\section{Acknowledgement}

Nil.

\section{References}

1. Australian Institute of Health and Welfare (2016) Australia's health 2016. Available at: https://www.aihw.gov.au/getmedia/c420f6f1-04644f43-b55a-62f995a0f8f3/ah16-3-6-stroke.pdf.aspx. [Accessed 2 Apr. 2018].

2. Chia NH, Leyden JM, Newbury J, Jannes J, Kleinig TJ (2016) Determining the number of Ischemic Strokes Potentially Eligible for Endovascular Thrombectomy: A Population-Based Study. Stroke 47: 1377-1380.

3. Saver JL, Goyal M, van der Lugt A, Menon BK, Majoie CB, et al. (2016) Time to Treatment with Endovascular Thrombectomy and Outcomes from Ischaemic Stroke: A Meta-Analysis. Journal of the American Medical Association 316(12): 1279-1288.

4. Australian Bureau of Statistics (2015) Southern Highlands and Shoalhaven (SA4) (114). Available at: http://stat.abs.gov.au. [Accessed: 13 May. 2018]

5. Stroke Foundation Australia (2017) National Stroke Audit - Acute Services Report 2017. Available at: https://strokefoundation.org.au/ What-we-do/Treatment.../Stroke-data.../Acute-audit. [Accessed: 13 May. 2018]

6. Albers GW, Bates VE, Clark WM, Bell R, Verro P, et al. (2000) Intravenous tissue-type plasminogen activator for treatment of acute stroke: The Standard Treatment with Alteplase to Reverse Stroke (STARS) Study. Journal of the American Medical Association 283: 1145-1150.

7. Romano JG, Muller N, Merino JG, Forteza AM, Koch S, et al. (2007) Inhospital delays to stroke thrombolysis: paradoxical effct of early arrival. Neurological Research 29: 664-666.

8. Robison T, Zaheer Z, Mistri AK (2011) Thrombolysis in Acute Ischemic Stroke: An Update. Therapeutic Advances in Chronic Disease 2(2): 119131.

9. Hacke W, Donnan G, Fieschi C, Kaste M, von Kummer R, Broderick JP, et al. (2004) Association of outcome with early stroke treatment: Pooled analysis of ATLANTIS, ECASS and NINDS rt-PA stroke trials. Lancet 363: 768-774.

10. Froehler MT, Saver JL, Zaidat O0, Jahan R, Aziz-Sultan MA (2017) Interhospital Transfer Before Thrombectomy Is Associated with Delayed 
Treatment and Worse Outcomes in the STRATIS Registry. Circulation 136(24): 2311-2321.
11. Goyal M (2016) Effects on Outcome in Endovascular Treatment of Acute Ischemic stroke: Results from the SWIFT PRIME Randomized Controlled Trial. Radiology 279(3). 